\section{Reverse Takotsubo Cardiomyopathy (rTTC) After a Severe Episode of Almond-Dependent, Exercise- Induced Anaphylaxis}

Betancor $D^{1}$, Nuñez-Borque E ${ }^{1}$, Esteban $\mathrm{V}^{1,2}$, Bueno-Díaz $\mathrm{C}^{2,3}$, de las Heras Gozalo $\mathrm{M}^{1,2}$, Pastor-Vargas $\mathrm{C}^{1,2 *}$, Cuesta-Herranz $\mathrm{J}^{1,2 *}$ ${ }^{1}$ Department of Allergy and Immunology, FIIS-Fundación Jiménez Díaz, UAM, Madrid, Spain

${ }^{2}$ RETIC ARADyAL, Instituto de Salud Carlos III, Spain

${ }^{3}$ Department of Biochemistry and Molecular Biology, Universidad Complutense de Madrid, Madrid, Spain

*Both authors contributed equally

J Investig Allergol Clin Immunol 2021; Vol. 31(3): 253-254 doi: $10.18176 /$ jiaci.0598

Key words: 7S globulin. Almond allergen. Almond-dependent, exerciseinduced anaphylaxis. Reverse takotsubo cardiomyopathy.

Palabras clave: Globulina 7S. Alérgenos de almendra. Anafilaxia por almendra inducida por ejercicio. Cardiomiopatía Tako-Tsubo reversible.

Nut allergy is one of the most frequent causes of anaphylaxis, often eliciting life-threatening reactions.

We report a case of reverse takotsubo cardiomyopathy (rTTC) in a patient after an episode of severe, almond-dependent, exercise-induced anaphylaxis in which a $7 \mathrm{~S}$ globulin (vicilin) was identified as the sensitizing allergen. rTTC is a rare variant of classic takotsubo cardiomyopathy (TTC) that has a different clinical profile and requires specific hemodynamic support.

A 28-year-old woman with no previous personal history of atopy experienced an episode of generalized urticaria, edema of the lips and hands, shortness of breath, and loss of consciousness after running for 30 minutes. She was admitted to the emergency department with cardiogenic shock. Her troponin I level was mildly elevated $(2.7 \mathrm{ng} / \mathrm{mL}$ [normal, $<0.4 \mathrm{ng} / \mathrm{mL}$ ) with anterior ST-segment (V1-V4) elevation on ECG and left ventricular dysfunction on transthoracic echocardiography (ejection fraction, 10\%-15\%), with basal and mid segment hypokinesis. She received intramuscular adrenaline and intravenous antihistamine, noradrenaline, and dobutamine, and her symptoms resolved. During admission, the coronary computed tomography scan was normal, magnetic resonance imaging revealed no myocardial edema or scarring, and left ventricular ejection fraction had gradually recovered to $60 \%$. Therefore, after evaluation by the Cardiology Department, the patient fulfilled the diagnosis criteria for rTTC [1] and was sent to the Allergy Department for evaluation. Written consent for publication was obtained from the patient.
A detailed clinical history looking for possible etiological agents (allergens) revealed that the reaction happened early in the morning and, prior to going running, she had only eaten almonds with no other food or drugs. Skin prick tests (SPTs) were performed (prick-by-prick) with nuts (almond, hazelnut, peanut, chestnut, sunflower seed, pine nut, walnut, pistachio, and cashew). SPT was also carried out with Betula verrucosa pollen (ALK, Spain) and purified protein (Pru p 3 [nonspecific lipid transfer protein, LTP]) (Roxal). SPT was positive to almond $(4 \mathrm{~mm})$ and hazelnut $(10 \mathrm{~mm})$ and negative for the remaining allergens.

The baseline tryptase value was normal $(3.48 \mu \mathrm{g} / \mathrm{L})$. Specific IgE (ImmunoCAP, Thermo Fisher Scientific) was positive for almond $(0.70 \mathrm{kU} / \mathrm{L})$ and hazelnut $(1.23 \mathrm{kU} / \mathrm{L})$ and negative for peanut, chestnut, walnut, pistachio, cashew, sunflower seed, and pine nut, as well as for purified allergens: LTP (Ara h 9, Cor a 8, and rPru p 3), PR-10 (rAra h 8), profilin (Pru p 4), gluten, rAra h 1 (7S globulin), rAra h 2 (2S albumin), and rAra h 3 (11S globulin).

In vitro experiments were performed with walnut, almond, and hazelnut extracts (Roxal). The protein concentration was adjusted to $1 \mathrm{mg} / \mathrm{mL}$. An immunoblotting assay performed as previously reported [2] revealed a 49-kDa IgE-binding band in the almond extract and 3 bands in hazelnut $(60 \mathrm{kDa}$, $49 \mathrm{kDa}$, and $14 \mathrm{kDa}$ ) (Figure, A). Immunoblotting-inhibition (preincubated with almond or hazelnut extract) revealed that the $49-\mathrm{kDa}$ band in hazelnut was inhibited by the almond extract (Figure, B). A 49-kDa band from the almond extract and a 14-kDa band from the hazelnut extract were extracted from the gel and identified by mass spectrometry, as previously reported [2]. Research conducted with protein databases identified the $49-\mathrm{kDa}$ protein as a vicilin and the $14-\mathrm{kDa}$

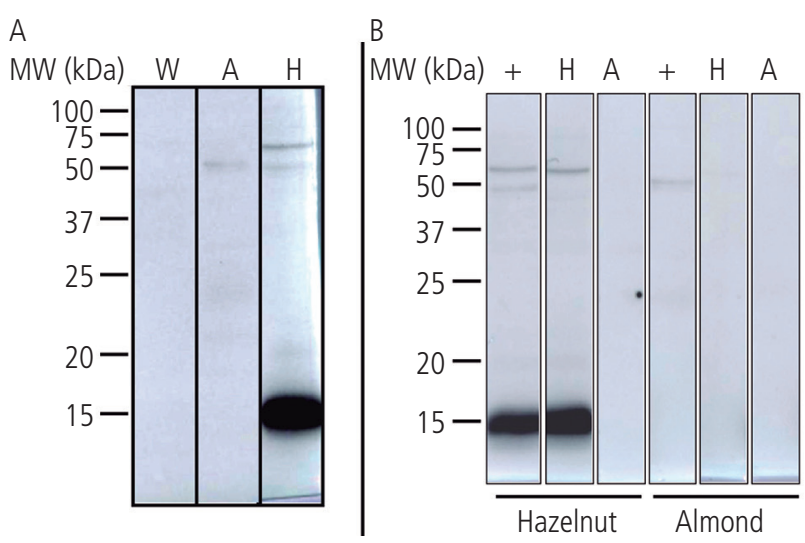

Figure. $A$, Detection of IgE-binding proteins in walnut $(W)$, hazelnut $(H)$, and almond (A) extract. B, Immunoblotting-inhibition results. Lane +, Patient's serum; Lane A, Patient's serum inhibited with almond extract; Lane $\mathrm{H}$, Patient's serum inhibited with hazelnut extract. MW indicates molecular weight marker. 
band as a $2 \mathrm{~S}$ albumin. Vicilins (7S globulin) are seed storage proteins from plants (seeds, nuts, and legumes). Although several 7S globulins have been reported as nut allergens, we should emphasize that this is the first time a $7 \mathrm{~S}$ globulin from almond has been identified as an allergen.

The patient avoided almond after experiencing the allergic reaction, although she continued eating other nuts without experiencing adverse reactions. She tolerated exercise (running) well. A challenge with almond was not performed owing to the severity of the reaction and sensitization to almond. Although hazelnut was tolerated, we recommended that the patient avoid both almond and hazelnut in the future, especially prior to exercising. Autoinjectable adrenaline and rescue medication were also prescribed.

Based on these data, the diagnosis was rTTC after a severe episode of almond-dependent, exercise-induced anaphylaxis, in which the etiological agent was a $7 \mathrm{~S}$ globulin (vicilin).

TTC, which is also known as stress cardiomyopathy or broken heart syndrome, is an acute but often reversible left ventricular dysfunction [3] that is usually triggered by emotional or physical stress. Several variants of TTC have been described based on the regions of ventricular wall motion abnormality, including the midventricular and basal wall. rTTC is a rare variant, which presents with basal instead of apical ballooning. The Takosubo Registry showed that only $2.2 \%$ of TTC cases were rTTC [4].

Nut allergy is one of the most frequent causes of anaphylaxis [5]. The nuts eliciting allergy depend on consumption habits, geographical region, environmental allergens, and patient age [6,7], and the severity of the reaction has been linked to the purified allergen involved in the reaction [8]. Walnuts and hazelnuts were the most frequent nuts eliciting allergy in Spain [7], even though almonds were the second most consumed nuts [9].

Food-dependent, exercise-induced anaphylaxis is a severe allergic reaction in which symptoms develop if exercise takes place within a few hours of eating a sensitizing food. The patient had eaten almonds 30 minutes before developing an anaphylactic reaction, and sensitization to almond was shown by both positive prick-prick test and specific IgE results. Immunoblotting revealed a 49-kDa IgE-binding band; this was identified as an almond vicilin (7S globulin), which appears to be the only purified allergen involved as an etiological agent. In this sense, we must highlight that seed storage proteins have been linked to the most severe allergic reactions [8].

Curiously, the patient was also sensitized to hazelnut (7S globulin and 2S albumin). 7S globulin from hazelnut was cross-reactive with its almond homolog, although this was not demonstrated for the $2 \mathrm{~S}$ albumin. Despite sensitization to both proteins in hazelnut, the patient ate hazelnut without experiencing adverse reactions. The patient tolerates exercise (running) well.

In summary, this is the first report of a $7 \mathrm{~S}$ globulin (vicilin) being identified as a new almond allergen. The allergen elicited a life-threatening reaction, namely, severe exercise-induced anaphylaxis complicated by rTTC.

\section{Acknowledgments}

The authors wish to thank Oliver Shaw for editing the paper for matters concerning English usage, flow, and style.

\section{Funding}

This work was supported by grants from the Instituto de Salud Carlos III and the thematic network and cooperative research centers ARADyAL RD16/0006/0013 and RD16/0006/0014, cofunded by FEDER grants.

\section{Conflicts of Interest}

The authors declare that they have no conflicts of interest.

\section{References}

1. Kotsiou OS, Xirogiannis KI, Gourgoulianis KI. Kounis Syndrome: Is it Really a Takotsubo-Like Syndrome? I Invest Allergol Clin Immunol. 2017;27(3):198-200.

2. Pastor C, Cuesta-Herranz J, Cases B, Perez-Gordo M, Figueredo $E$, de las Heras $M$, et al. Identification of major allergens in watermelon. Int Arch Allergy Immunol. 2009;149:289-90.

3. Awad HH, McNeal AR, Goyal H. Reverse Takotsubo cardiomyopathy: a comprehensive review. Ann Transl Med. 2018:6:460.

4. Ghadri JR, Cammann VL, Napp LC, Jurisic S, Diekmann J, Bataiosu DR, et al; International Takotsubo (InterTAK) Registry. Differences in the Clinical Profile and Outcomes of Typical and Atypical Takotsubo Syndrome: Data From the International Takotsubo Registry. JAMA Cardiol. 2016;1:335-40.

5. Pouessel G, Turner PJ, Worm M, Cardona V, Deschildre A, Beaudouin $E$, et al. Food-induced fatal anaphylaxis: From epidemiological data to general prevention strategies. Clin Exp Allergy. 2018;48:1584-93.

6. Vereda A, van Hage M, Ahlstedt S, Ibañez MD, Cuesta-Herranz J, van Odijk J, et al. Peanut allergy: Clinical and immunologic differences among patients from 3 different geographic regions. J Allergy Clin Immunol. 2011;127:603-7.

7. Haroun-Díaz E, Azofra J, González-Mancebo E, de Las Heras M, Pastor-Vargas C, Esteban V, et al. Nut allergy in two different areas of Spain: Differences in clinical and molecular pattern. Nutrients. 2017:9:909.

8. Canonica GW, Ansotegui IJ, Pawankar R, Schmid-Grendelmeier $P$, van Hage $M$, Baena-Cagnani CE, et al. A WAO - ARIA $\mathrm{GA}^{2} \mathrm{LEN}$ consensus document on molecular-based allergy diagnostics. World Allergy Organ J. 2013;6:1-17.

9. Aranceta J, Pérez Rodrigo C, Naska A, Vadillo VR, Trichopoulou A. Nut consumption in Spain and other countries. Br J Nutr. 2006;96 (Suppl 2):S3-11.

Manuscript received May 15, 2020; accepted for publication July 24, 2020.

Diana Betancor

Avda. Reyes Católicos, 2

Madrid, Spain

E-mail: diana13_b@hotmail.com 